\title{
Effekte und Wirkmechanismen der so genannten Abhärtung
}

\author{
Rainer Brenke \\ Abteilung für Naturheilverfahren, Hufeland-Klinik, Bad Ems, Deutschland
}

Schlüsselwörter

Abhärtung · Kaltreize · Eisbaden · Sauna · Prävention

\section{Zusammenfassung}

Thermische Reize, insbesondere Kaltreize zum Zweck der Prophylaxe und Therapie, werden in unserer «aktiven» Zeit vernachlässigt. Sie können aber nicht nur bei vielen Krankheiten zum Einsatz kommen, sondern auch auf unterschiedlichen Wegen prophylaktisch im Sinne einer Abhärtung wirken. Dabei wird das Immunsystem oftmals in den Vordergrund der Betrachtungen gestellt, bildet aber nur einen Mosaikbaustein im Spektrum der unterschiedlichen Wirkmechanismen. Die Thermotherapie bzw. Warm- und Kaltreize sollten daher gezielter und konsequenter zur Prophylaxe und Therapie eingesetzt werden.

\section{Einleitung}

Der Gedanke der «Abhärtung» ist ein klassisches naturheilkundliches Thema und wird von der konventionellen Medizin weitgehend ignoriert. Vielleicht liegt das auch daran, dass der Begriff über Jahrhunderte hinweg nicht nur medizinisch besetzt war und auch im Dienste verschiedener Ideologien stand. Die weitgehende Ignorierung dieses wichtigen Themas geht aber an der Tatsache vorbei, dass die Abhärtung wie kaum ein anderes naturheilkundliches Thema inzwischen auf vielfältige Weise wissenschaftlich durchdrungen wurde. Der vorliegende Beitrag soll einen Überblick über wichtige Erkenntnisse zum Nachweis und zu den Wirkmechanismen der Abhärtung geben.

\author{
Key Words \\ Inurement $\cdot$ Cold stimuli $\cdot$ Ice bathing $\cdot$ Sauna $\cdot$ Prevention
}

\section{Summary}

Effects and Modes of Action of the So-Called Inurement

Thermal stimuli, particularly cold stimuli for prevention and therapy purposes, are neglected in the 'active' modern times. However, they can not only be helpful regarding many illnesses but can also be efficient for preventive purposes such as inurement. In this regard, the immune system is often placed in the foreground of the examinations but it represents only a small part of the different modes of action. Thermotherapy or warm and cold stimuli, respectively, should therefore be applied more purposefully as well as more consequently for prevention and therapy.

\section{Abhärtung: Versuch einer zeitgemässen Definition}

Nach dem Lexikon der Medizin versteht man heute unter Abhärtung «durch klima- und hydrotherapeutische Kaltreize ausgelöste funktionelle Adaptation, die über eine verbesserte Thermoregulation und unspezifische Abwehr die Empfindlichkeit gegenüber Erkältungskrankheiten vermindert und die physische Leistungsfähigkeit des menschlichen Organismus steigert» [1]. Es erfolgt hier eine Beschränkung auf Kaltreize; Abhärtung kann jedoch auch durch andere Reize zustande kommen.

«Abhärtung» hat als Begriff aber eine lange Geschichte und war bereits im 18. Jahrhundert verbreitet. Inhaltlich wurde Abhärtung umfassender als heute gesehen und hatte auch die Bedeutung im Sinne von Erziehung zu

\section{KARGER}

Fax +49761 4520714
(๑) 2010 S. Karger GmbH, Freiburg

www.karger.com/sz 
allgemeiner körperlicher Härte und Unempfindlichkeit. Schon damals sah man in der Abhärtung ein notwendiges Gegenmittel gegen eine «Verweichlichung» der Menschen in der modernen Zivilisationsgesellschaft. Psychologische Faktoren sind zwar schwer erfassbar, werden aber - wie eine geringere Stressanfälligkeit - einen wesentlichen Anteil an der Gesundheitsstabilisierung durch Abhärtung haben.

Auch in Anlehnung an die alte Tradition möchten wir heute Abhärtung als die "Folge einer wiederholten bewussten oder unbewussten Exposition des Menschen gegenüber natürlichen Reizen mit einer allgemeinen Erhöhung der Widerstandsfähigkeit gegenüber Krankheiten» definieren [2]. Bei dem heutigen Bestreben nach einer evidenzbasierten Medizin gestaltet sich der Wirksamkeitsnachweis der Abhärtung problematisch, da abhärtende Massnahmen meist beim (noch) Gesunden eingesetzt werden. Wie weist man nun nach, dass der Gesunde noch gesünder wird? Dies ist nur durch eine umfassende $\mathrm{Zu}-$ sammenschau von experimentellen, klinischen und epidemiologischen Daten möglich, da Labormethoden alleine schlecht geeignet sind, denn sie dienen in der Regel dem Nachweis von Krankheiten und nicht der Messung der gesundheitlichen Stabilität. Nimmt man die Häufigkeit einfacher grippaler Infekte als Massstab, so spielt die Psyche für die subjektiv empfundene gesundheitliche Stabilität eine grosse Rolle, was epidemiologische Angaben verfälscht, denn der Abgehärtete hält sich ja auch für stabiler und wird weniger zum Arbeitsausfall neigen als der Nicht-Abgehärtete. Daraus folgert, dass nur alle Daten zusammen eine sichere Aussage zulassen.

\section{Abhärtung durch Kaltreize}

Abhärtung ist auch heute noch gedanklich untrennbar mit Kaltreizen verbunden. Wirksamkeit und Wirkungsweise konnten in den letzten Jahren an einer Reihe von Studien nachgewiesen werden. In der Praxis kommen heute besonders kaltes Duschen, Wassertreten, kalte Armbäder, aber auch das klassische Tautreten oder als Extremvariante das Winterbaden zur Anwendung. Nach neueren Untersuchungen scheinen allerdings einfache hyd rotherapeutische Anwendungen zumindest im Vorschulalter keinen Einfluss auf die Infekthäufigkeit und deren Verlauf zu haben [3]. Dem stehen frühere, klinisch positive Erfahrungen entgegen [4-6]. Bei Erwachsenen scheinen aber auf jeden Fall auch einfache kalte Güsse einen nachweisbaren Effekt im Hinblick auf die Häufigkeit grippaler Infekte zu haben, wobei ein statistisch signifikanter Einfluss ähnlich wie bei der Sauna nach einem Vierteljahr nachweisbar ist [7]. Auch Goedsche und Mitarbeiter konnten 2007 zeigen, dass eine Serie von kalten Güssen (Obergüsse nach Kneipp) das subjektive Befinden und die Häufigkeit von Atemwegsinfek- tionen bei Patienten mit chronisch obstruktiver Bronchitis (COPD) günstig beeinflusst [8].

Mit sehr kalten Luftbädern am Meeresstrand im Winter hat Menger bereits $1980 \mathrm{sehr}$ gute Erfahrungen bei chronisch kranken Kindern gemacht [9]. Über positive Effekte auf die Lungenfunktion hat er auch 1990 berichtet, wobei die Erfahrungen bei 40 Mädchen im Alter von 10-16 Jahren mit Asthma bronchiale vorgestellt wurden. Während eines Kaltluftbades an der Nordsee zwischen Dezember und April mit Lufttemperaturen zwischen -10 und $+10^{\circ} \mathrm{C}$ kam es anfangs zwar in Abhängigkeit von der Aussentemperatur in rund der Hälfte der Fälle zu einem Bronchospasmus, der sich jedoch während des kalten Luftbades zurückbildete. Menger interpretierte diese Beobachtung als Ausdruck einer sympathikotonen Stimulation [10].

Dass seriell angewandte Kaltreize mehr vermögen, als nur die Infekthäufigkeit zu senken, zeigten Jacob und Volger [11] erst kürzlich nochmals an Reha-Patienten, die sich unter anderem wegen eines Hypertonus einer stationären Rehabilitationsmassnahme unterzogen und in diesem Zusammenhang 5-12 hydrotherapeutische Anwendungen pro Woche erhielten (Kniegüsse, Schenkelgüsse, Armgüsse kalt oder wechselwarm, abendliches Wassertreten). Die Blutdrucksenkung war in dieser HydrotherapieGruppe, die ihre Anwendungen zusätzlich zu den übrigen typischen Reha-Massnahmen erhielt, stärker ausgeprägt als in der Kontrollgruppe. Auch der Blutdruckanstieg unter Belastung fiel geringer aus; die Steigerung der Leistungsfähigkeit war grösser. Eine anfängliche antihypertensive Medikation konnte auch nur in der HydrotherapieGruppe reduziert werden [11].

Eine extreme Variante der Abhärtung durch Kaltreize, die die von Priessnitz empfohlenen Methoden übertrifft, stellt das Winterschwimmen oder das Eisbaden dar. Hier erfolgt im Winter nach einem kurzen Erwärmungslauf ein kurzes Bad im freien Gewässer bis zu wenigen Minuten Dauer. Winterbader geben neben einer allgemeinen Steigerung des gesundheitlichen Wohlbefindens vor allem eine gesenkte Infektneigung an, die sich auch nachweisen lässt. Binnen 5 Jahren halbiert sich die Zahl der Arztkonsultationen wegen grippaler Infekte [12,13]. Die Belastung des Herz-Kreislauf-Systems ist allerdings deutlich höher als bei der Sauna. Ein arterieller Hypertonus, die periphere arterielle Verschlusskrankheit (paVK), Herzrhythmusstörungen sowie alle Erkrankungen, die sich durch Kälte verschlechtern, verbieten diese Massnahme.

\section{Sauna als effektivste Methode zur Abhärtung}

Die Sauna ist sicher die effektivste und praktikabelste Methode zur Abhärtung. Sie war zu Zeiten der Protagonisten des Abhärtungsgedankens wie Kneipp und Priessnitz noch kaum verbreitet und hat einen deutlichen Auf- 
schwung in Deutschland erst ab Mitte des vergangenen Jahrhunderts erfahren. Für die Sauna konnte für das Kindesalter schon vor längerer Zeit ein eindeutiger präventiver Effekt auf die Häufigkeit von Erkältungskrankheiten gezeigt werden [14-16]. Hinsichtlich der Schulfehltage hat man einen deutlichen Effekt nach 3 Monaten regelmässigen Saunabesuchs feststellen können. Die Zahl der Schulfehltage wegen Infekten sinkt zeitweilig auf Null [17]. Einer der Gründe scheint eine verbesserte lokale Abwehr an den Schleimhäuten zu sein [18].

Auch für das Erwachsenenalter sind die Zahlen zum Einfluss der Sauna eindeutig. 27\% befragter regelmässiger Saunabesucher gaben an, «völlig frei», 56\% «fast frei» von Infekten zu sein. Bei der «asiatischen Grippe», einer Pandemie im Jahr 1957, erkrankten nur 5\% der Saunabesucher, jedoch 16-47\% der übrigen Beschäftigten unterschiedlicher Betriebe. Allgemein findet man immer wieder die Angaben, dass Häufigkeit und Arbeitsunfähigkeitsdauer bei grippalen Infekten reduziert sind [19-22].

\section{Weitere Methoden zur Abhärtung}

Es gibt eine Reihe weiterer Empfehlungen zur Abhärtung, die an dieser Stelle erwähnt werden sollen, obwohl es für sie nur wenige wissenschaftliche Belege sowohl im Hinblick auf ihre Effektivität als auch auf ihre mögliche Wirkungsweise gibt. Bei der erheblichen Bedeutung, die der breite Einsatz abhärtender Methoden im Hinblick auf die Gesundheit der Bevölkerung jedoch haben könnte, sollen sie an dieser Stelle dennoch besprochen werden, wobei man ihre Erwähnung auch als Aufforderung zur erneuten und vertieften wissenschaftlichen Beschäftigung mit diesem Thema ansehen kann.

\section{Lokale Abhärtung}

Eine Methode zur örtlichen Abhärtung der Schleimhäute stellt die so genannte Schleimhautregie nach Vogler dar. Vogler war Mitte des 20. Jahrhunderts Ordinarius für Physiotherapie an der Berliner Charité. Auch wenn es zu diesem Thema nur wenige Untersuchungen gibt, erscheint das Verfahren sinnvoll, wenn man die reflektorischen $\mathrm{Zu}-$ sammenhänge zwischen den Schleimhäuten des NasenRachen-Raums und den oberen Luftwegen bedenkt (Stichwort: «Sinubronchiales Syndrom»). Zur Schleimhautregie zählen folgende Schritte:

- Phase 1: Abräuspern aus Larynx und Pharynx (im Original zusätzlich beschrieben: Andampfung und Nasentamponade, wobei letztere aber aus meiner Sicht nicht in die Hand des Patienten gehört);

- Phase 2: Bürsten von Zunge (Vorsicht!), Zahnfleisch und Mundschleimhäuten;
- Phase 3: Gurgeln;

- Phase 4: Gesichtsgüsse;

- zusätzlich (nicht bei Vogler): Nasenspülungen mit isotonischer Kochsalzlösung, zum Beispiel mit Emser Salz. Alleine der mechanische Reiz des Gurgelns, Räusperns und sanften Bürstens der Schleimhäute wird lokal die Durchblutung steigern und damit die Abwehrsituation verbessern. Nasenspülungen mit Emser Salzlösungen («Spülbirne», in der Apotheke erhältlich) sollen gegenüber üblichen Kochsalzlösungen Vorteile haben. Vom Trend her fand Bachmann einen besseren Effekt von Emser Salzlösung gegenüber $\mathrm{NaCl}$-Lösung bei chronischen Nebenhöhlenentzündungen (Bachmann et al., 2000, zitiert nach Fa. Siemens, Bad Ems). Keinesfalls geeignet sind jedoch Spülungen mit reinem Leitungswasser, da es hier wiederholt zu einer Otitis media gekommen ist, was man bei isotonischer Emser Salzlösung nicht fand. Bei regelmässiger Anwendung konnte nach einer Woche eine signifikante Beschleunigung der Saccharin-Clearance für die Emser Salzlösung beschrieben werden (Wendeler et al., 1997, zitiert nach Fa. Siemens, Bad Ems). Ausserdem kam es zu einer subjektiven Beschwerdelinderung und einer Verbesserung der Nasenatmung.

\section{Abhärtung im weiteren Sinne}

Ein näheres Eingehen auf die auch schon zu Zeiten von Kneipp und Priessnitz popularisierten Methoden der körperlichen Bewegung würde den Rahmen dieses Beitrags sprengen. Bewegung als «Gesundheitsmotor» ist unbestritten, auch wenn der Trend in Richtung eines zunehmend moderaten, nicht übertriebenen Trainings geht. Auch hier hat die oft zitierte Regel, dass zu starke Reize hemmend wirken, zu geringe dagegen keine Wirkung entfalten, ihre Gültigkeit. Die Auffassungen, was eine optimale und adäquate Dosis ist, unterliegen jedoch in der letzten Zeit einem Wandel, und der Trend geht in Richtung eher milderer Reize (z.B. $2 \times 30 \mathrm{~min}$ Sport/Woche). Dabei kommt es natürlich auch auf das jeweilige Ziel an, das mit der sportlichen Aktivität verfolgt wird. Noch hat in den allgemeinen Empfehlungen ein Ausdauersport Vorrang, jedoch stellt sich mehr und mehr heraus, dass auch ein sinnvoll und gut angeleitetes Krafttraining viele gesundheitliche Vorteile bringt, die weit über den Kraftzuwachs hinausgehen. Hier sollte auf individuelle Präferenzen geachtet werden - nur das, was man mit hoher Motivation und positiver Emotion betreibt, wird letztlich auch von gesundheitlichem Nutzen sein. Zumindest für sportliche Aktivitäten gibt es bereits Belege, die einen Zusammenhang zwischen Motivation und erzielter Leistung aufzeigen $[23,24]$.

Ähnliches gilt für eine dosierte, individuell angepasste Sonnenexposition. Bei dem Hang zur Übertreibung und

Schweiz Z Ganzheitsmed 2010;22:37-44 
Überdosierung in den letzten Jahrzehnten (Solarien, vermehrter UV-Einfluss durch das Ozonloch, Emanzipation durch «freizügige» Kleidung) konnten negative Effekte wie ein vermehrtes Auftreten von Hautkrebs zumindest in der Retrospektive und mit dem Wissen von heute kaum ausbleiben. Es besteht aber die Gefahr, dass das Verhalten jetzt in das andere Extrem umschlägt und die vielfältigen positiven Effekte von Licht und auch UV-Strahlung in Vergessenheit geraten. Als Beispiele sollen positive Effekte von Licht auf die Psyche des Menschen, der Schutz durch UVEinwirkung vor Osteoporose, die Anregung des Immunsystems, positive Effekte auf das Herz-Kreislauf-System und vielleicht auch ein gewisser protektiver Effekt bezüglich mancher maligner Erkrankungen an inneren Organen genannt werden. Wie so oft liegt auch hier der Schlüssel zur Gesundheitsförderung in der Dosis, die es individuell zum Beispiel anhand des Hauttyps herauszufinden gilt. Eine gute Zusammenfassung über die positiven Effekte des Klimas allgemein und der Sonnenstrahlung im Besonderen gab Angela Schuh bereits 1998 [25].

Als abhärtende Massnahme, die ebenfalls etwas mit thermischen Reizen zu tun hat, kann man insbesondere bei Kindern auch die Wahl einer geeigneten Kleidung ansehen. Etliche nur wenig luftdurchlässige Kleidungsstücke wie ältere Anoraks erzeugen am Körper ein Mikroklima, das dem der Subtropen entspricht. Ein Wärmestau und Schwitzen mit Durchfeuchten der Kleidung sind daher bei körperlichen Aktivitäten unvermeidbar. Danach kann es durch Verdunstungskälte leicht zur Unterkühlung kommen. Die natürlichen «Gesundheitsmotoren» wie Luft und dosierte Kaltreize sollten die Möglichkeit haben, an die Haut zu gelangen. Mehrere dünne Kleidungsstücke, von denen bei Bedarf auch eines ausgezogen werden kann, übereinander gezogen («Zwiebel-Look») sind daher besser als ein dickes Kleidungsstück [4, 5].

Im weiteren Sinne kann man zu den abhärtenden, die Abwehr des Organismus stabilisierenden Massnahmen auch eine Vollwerternährung rechnen, da der Darm unser wichtigstes Immunorgan ist. Ausserdem wirkt die bedarfsgerechte Zufuhr von Vitaminen und anderen Antioxydantien abwehrsteigernd.

\section{Wirkmechanismen der Abhärtung}

\section{Thermoregulatorische Anpassungen}

Für die Sauna konnte mithilfe eines Tests zur akralen Wiedererwärmung nach einem standardisierten Kaltreiz langfristig eine eindeutige Verbesserung der Durchblutungsregulation der Haut an den Fingern gezeigt werden $[26,27]$. Unmittelbar nach einem Saunabesuch verbessern sich so- wohl Haut- als auch Muskeldurchblutung [28]. Wichtiger sind jedoch die langfristigen Durchblutungsänderungen und hier speziell die der Akren (Hände). Da die Akren auf nervalem Weg mit der Durchblutung im Nasen-RachenRaum zusammenhängen, ist von einer verbesserten Durchblutungsregulation auch an den Schleimhäuten und damit von einer verbesserten Abwehrlage auszugehen. Die Durchblutung im Nasen-Rachen-Raum ist wahrscheinlich viel entscheidender für die Infektanfälligkeit als manche immunologischen Befunde, denn immunologische Abwehrmechanismen im Blut können nur dann besonders gut wirken, wenn sie durch eine gute Durchblutung auch den Ort des Geschehens erreichen.

Auch für wiederholte Kaltreize wie beim Winterbaden fanden wir deutliche thermoregulatorische Anpassungen an der akralen Durchblutungsregulation [29]. Hier zeigten Eisbader auch bei kalter Umgebungstemperatur im Winter eine höhere Hautdurchblutung und schnellere Wiedererwärmung an den Fingern als nicht angepasste Probanden. Es ist davon auszugehen, dass die bessere Durchblutung der Finger reflektorisch auch hier mit einer besseren Durchblutung im Nasen-Rachen-Raum und damit einer gesteigerten Infektabwehr einhergeht. Lange Andersen [30] wies bereits 1960 darauf hin, dass bei kälteadaptierten Menschen die Hautdurchblutung bei niedrigen Umgebungstemperaturen besser ist als bei nicht akklimatisierten Probanden. Unabhängig von der besseren Infektabwehr mag dies vom Standpunkt der Thermoregulation zunächst wenig sinnvoll erscheinen, denn der Wärmeverlust ist bei kalter Umgebung höher. Vom Gesamtorganismus her betrachtet ist diese Reaktion aber durchaus sinnvoll, denn die Gefahr lokaler Frostschäden ist geringer und die Arbeitsfähigkeit sowie Geschicklichkeit besonders der Hände in Kälte sind verbessert. Verbunden ist mit diesen Anpassungen der Hautdurchblutung meist ein Absinken der Körperkerntemperatur.

Die thermoregulatorischen Anpassungen haben nach neueren Erkenntnissen offenbar eine viel grössere Bedeutung als bisher angenommen. Eindeutig sind bei regelmässigem Saunabesuch eine bessere Durchblutung der Haut, ein vermehrtes Schwitzen bei Wärmeeinwirkung, eine schnellere Rückkehr der in Wärme erhöhten Kerntemperatur zu den Ausgangswerten und ein Abfall der Ruhewerte der Körperkerntemperatur zu beobachten [31, 32]. Eine geringere Kerntemperatur ist aber statistisch mit einer längeren Lebenserwartung verbunden [33]. Durch thermische Reizserien wie die Sauna kann bereits nach Wochen die Kerntemperatur um rund $0,5{ }^{\circ} \mathrm{C}$ sinken. Dies hätte rein statistisch eine Steigerung der Lebenserwartung um rund 5 Jahre zur Folge. Würde man mit den thermischen Anpassungen im mittleren Lebensalter beginnen, blieben immer noch 2-3 Jahre [34]. 


\section{Abhärtung und das vegetative Nervensystem}

Postuliert wird immer wieder der Einfluss thermischer Reizserien auf das vegetative Nervensystem im Sinne einer «Vagotonisierung» oder «Umstimmung» in Richtung Trophotropie. Da sich der Begriff der «Vagotonisierung» sowohl in der medizinischen Literatur als auch in der Umgangssprache durchgesetzt hat, soll er an dieser Stelle beibehalten werden, obwohl in korrekter Weise von einer «Parasympathikotonisierung» gesprochen werden müsste, da der Gegenspieler des Sympathikus der Parasympathikus ist und der N. vagus nur einen Teil des parasympathischen Nervensystems darstellt. Die Belege für eine «vegetative Umstimmung» sind jedoch - unabhängig von der korrekten Nomenklatur - insgesamt eher spärlich. Für ein Nachlassen des Sympathikus-Einflusses sprechen unter anderem eine gesenkte Vanillin-MandelsäureAusscheidung im Zusammenhang mit regelmässigem Saunabesuch sowie eine Änderung am vegetativen Herznerventonus, der sich in einer gesenkten Herzfrequenz bei gleichbleibender Sinusarrhythmie äussert. Auch dies ist im Sinne eines nachlassenden Sympathikus-Einflusses zu werten, zumindest wenn dieser von den Ausgangswerten her erhöht war [26]. Auch bei einer Serie von Kaltwasserbädern sinkt der Sympathikotonus - ebenso wie nach einer Serie von Bürsten- oder Schöpfbädern [35, 36]. Die oft postulierte «Vagotonisierung» bzw. «Parasympathikotonisierung» oder «Trophotropisierung» scheint demnach eher in einem Nachlassen eines krankhaft erhöhten Sympathikotonus und damit einem relativen Überwiegen des Parasympathikotonus als in einer direkten «Stärkung» des Parasympathikotonus zu bestehen.

Inhaltlich in eine gleiche Richtung weisen Untersuchungen an 28 Saunagängern und 9 Vergleichspersonen zur Konzentration von der Substanz P im Blutplasma. Es handelt sich hierbei um einen Neuropeptidtransmitter, der sich oft gegenläufig zu den Stresshormonen verhält und zum Beispiel auch an der Einschlafreaktion sowie an Schmerzbewältigungsmechanismen beteiligt ist. Bei den Saunagängern war deren Konzentration erhöht, was man in Zusammenhang mit einer verringerten Stressanfälligkeit bringen kann. Akut war nur ein Einfluss bei spätabendlichem Saunabesuch erkennbar [37]. Auch regelmässige Winterschwimmer weisen einen erhöhten Trend zu Substanz-P-Werten im Blutplasma und damit mutmasslich eine höhere vegetative Stabilität auf [38].

\section{Abhärtung und Immunsystem}

Allgemein bekannt ist der Anstieg der Leukozytenzahl im Blut nach Stress - so auch nach einer Sauna oder einem intensiven Kaltreiz. Die Zahl der Leukozyten sagt aber wenig über ihre Aktivität aus. Der Effekt ist vermutlich unspezifisch, bei vielen Reizen zu beobachten und sollte nicht überbewertet werden [39].

Bieger und Mitarbeiter berichteten bereits 1998 [40] über die Immunologie der Abhärtung im Zusammenhang mit einer 4-wöchigen Kneipp-Kur, bei der die hydrotherapeutischen Anwendungen im Wesentlichen aus einem Vollguss an jedem Morgen bestanden. Schon die einzelne Anwendung veränderte den Zustand des zellulären Immunsystems deutlich. Besondere Bedeutung bei den längerfristigen Anpassungen schien den CD4-Helferzellen zuzukommen, wobei die Interleukin(IL)-2-Rezeptorexpression kontinuierlich zunahm; die von Interferon(IFN)- $\gamma$ sank stetig. Die Autoren messen den zellulären Immunreaktionen im Zusammenhang mit der Abhärtung eine besondere Bedeutung bei; die unspezifische Immunabwehr scheint nach diesen Ergebnissen eher von untergeordneter Bedeutung zu sein.

Den positiven Effekt eines täglichen morgendlichen Wassertretens über 4 Wochen demonstrierten Kreutzfeld und Mitarbeiter 2003 [41]. Die Auswirkungen betrafen sowohl das Immunsystem als auch klinische Effekte. Zwar verringerte sich nicht die Erkrankungshäufigkeit, wohl aber sank deren Dauer und Schwere. Parallel dazu erfolgte ein Anstieg der Zahl von IFN- $\gamma$ - und IL-2-produzierenden T-Lymphozyten. Goedsche und Mitarbeiter demonstrierten 2007 [8], dass die intrazelluläre Expression von IL-4 durch T-Lymphozyten bei einer Serie kalter Obergüsse nach Kneipp bei Patienten mit COPD abfiel, während IFN- $\gamma$ anstieg.

Noch ausgeprägter und vor allem langfristig nachweisbar scheinen die immunologischen Veränderungen bei der Sauna zu sein. Als ein unspezifischer Parameter der Abwehr kann der Gehalt an IFN von Blut und Gewebe angesehen werden. Auch hier fanden sich die deutlichsten Ergebnisse im Zusammenhang mit der Sauna. IFN spielt sowohl eine Rolle bei der unspezifischen Abwehr (Immunmodulation) als auch bei der Krebsabwehr. Zwar kam es unmittelbar zu keinen Veränderungen, langfristig ist der Titer aber deutlich erhöht. Dies traf aber nur bei langjährigem und regelmässigem Saunabesuch zu, wobei die Sauna über das ganze Jahr wenigstens einmal wöchentlich besucht wurde [13, 34, 42, 43].

Die Zahl und Stimulierbarkeit der natürlichen Killerzellen (NK-Zellen) im Blut ist sowohl unmittelbar nach der Sauna als auch langfristig erhöht [44]. Allerdings können Teilmechanismen der Abwehr, wie zum Beispiel die Granulozytenaktivität, unmittelbar durch Sauna auch gehemmt werden, was einer der Gründe dafür ist, dass man mit einem akuten Infekt so intensive hydrotherapeutische Massnahmen wie die Sauna meiden sollte $[45,46]$. Als ein weiteres Indiz für eine unspezifische Aktivierung von $\mathrm{Ab}$ wehrmechanismen durch die Sauna kann der Abfall des 
Komplementfaktors C3 bei gleichbleibendem Faktor C4 gewertet werden [39].

Einen wesentlichen Beitrag zur lokalen Abwehr leistet auch die Funktion des Flimmerepithels der Schleimhäute. Zumindest für die Sauna konnte mithilfe der mukoziliären Clearance gezeigt werden, dass diese unspezifische Funktion der Abwehr unmittelbar nach der Sauna erhöht ist. Als Methode kam dabei ein Saccharintest zum Einsatz, wobei die Zeit bis zum Auftreten eines süssen Geschmacks einer mit der Nasenschleimhaut in Verbindung gebrachten Saccharintablette gemessen wird. Die verkürzte Zeit bis zum süssen Geschmack nach der Sauna gilt als Indiz für eine erhöhte mukoziliäre Aktivität und damit für eine verbesserte lokale Abwehr [47].

Inhaltlich vergleichbar ist der Anstieg von Immunglobulin A im Speichel, was auch bei Kindern beobachtet werden konnte [14, 48, 49]. Immunglobulin A spielt eine entscheidende Rolle als erste Immunbarriere an den Schleimhäuten; eine lokal erhöhte Konzentration macht das Anhaften von Viren oder Bakterien an den Schleimhäuten und damit eine Infektion unwahrscheinlicher.

\section{Abhärtung und Radikalmetabolismus}

Ein neuer Mechanismus der Abhärtung konnte bei der Untersuchung von Parametern des Radikalmetabolismus im Zusammenhang mit kurzen Kaltreizen wie kaltem Duschen oder Winterschwimmen und der Sauna aufgedeckt werden. Unmittelbar kommt es zu einer vermehrten Freisetzung freier Radikale, langfristig dagegen zu Anpassungen mit einer Stärkung antioxidativer Schutzmechanismen. Man könnte das als «Regimen refrigerans als dosierter oxidativer Stress» bezeichnen, dessen Anpassungsreaktionen die Gesundheit stabilisieren. Als Parameter dienten zum einen Malondialdehyd und 4-Hydroxynonenal im Blutplasma sowie die Konzentration von oxidiertem und reduziertem Glutathion in den Erythrozyten. Ausserdem fanden sich bei Eisbadern höhere Enzymaktivitäten bei den Enzymen Katalase, Superoxiddismutase und Glutathionperoxidase als weitere Hinweise auf die gesundheitsstabilisierende Wirkung abhärtender Massnahmen [29, 50-56]. Eine langfristige Verringerung der Belastung durch freie Radikale fand sich auch bei regelmässigem Saunabesuch [57].

Wärme und Kälte scheinen insgesamt aber differente Wirkungen zu haben. Die Anregung des Immunsystems ist offenbar an eine milde Hyperthermie, also die Wärme, gekoppelt, die Anregung der Durchblutung dafür an die Kälte bzw. den Wechselreiz zwischen Wärme und Kälte. Ebenso sind die vegetative Stabilisierung mit der «Parasympathikotonisierung» («Vagotonisierung») und die biochemischen Anpassungen (Stärkung antioxidativer
Schutzsysteme) mit den Kaltreizen verknüpft. Auch wenn wir die Sauna als die wohl effektivste Methode zur Abhärtung ansehen, kommt man nicht um die Schlussfolgerung herum, dass die alte Auffassung, nach der Abhärtung etwas mit der Anpassung an Kaltreize zu tun hat, durchaus berechtigt ist. Dabei sollte nicht vergessen werden, dass das Wesen der Sauna in dem thermischen Wechselreiz von Wärme und Kälte besteht. Ohne zwischengeschaltete Kaltreize hat man es nicht mit einer Sauna zu tun, und ganz allgemein führen serielle Anwendungen mit ausschliesslichen Wärmereizen nicht zu einer Verbesserung der Durchblutungsregulation [58].

\section{Wirkungswege und Methoden der Abhärtung im Überblick}

Als Wirkungswege der Abhärtung kommen aus heutiger Sicht neben psychologischen Beeinflussungen folgende physiologische Anpassungen in Betracht [59]:

- Verbesserung der Thermoregulation und damit verbundene Kreislaufumstellungen (bessere Durchblutung der Akren);

- reflektorische Beeinflussung der Schleimhautdurchblutung im Nasen-Rachen-Raum;

- vegetative Stabilisierung mit Abnahme eines Hypersympathikotonus (als «Parasympathikotonisierung» oder gewöhnlich auch als «Vagotonisierung» bezeichnet);

- Beeinflussung von Immun- und Resistenzparametern (wahrscheinlich in erster Linie an eine milde Hyperthermie gekoppelt);

- biochemische Veränderungen im Sinne einer Stärkung antioxidativer Schutzmechanismen (das Regimen refrigerans als «oxidativer Stress»). Dieser Effekt scheint an die Kaltreize gekoppelt zu sein.

Als Methoden können zur Abhärtung aus heutiger Sicht empfohlen werden:

- Regelmässiger Saunabesuch (mit Kaltreizen!) 1- bis 2-mal/Woche;

- morgendliches kaltes Duschen;

- Wassertreten, kalte Güsse oder Wechselbäder für die Unterschenkel (einschliesslich solcher Varianten wie z.B. Tautreten);

- Winterschwimmen 1-mal/Woche;

- dosiertes körperliches Ausdauertraining;

- individuell angepasste Sonneneinwirkung;

- gesunde, vitaminreiche Vollwerternährung (Vitamine A, C und E als Antioxidantien, ausserdem Stärkung des Immunsystems im Darm);

- «vernünftige» Kleidung;

- «Schleimhautregie».

Bei der Auswahl bzw. Empfehlung der Methoden sollten stets Alter, Konstitution und Gesundheitszustand beachtet 
werden. Vernünftige Kleidung und Vollwerternährung können natürlich jedem empfohlen werden. Die Sauna setzt eine Leistungsfähigkeit von mindestens 60-75 Watt voraus; für das Winterschwimmen ist von einer höheren Belastung auszugehen. Intensive Kaltreize verbieten sich bei allen asthenischen Personen oder solchen, die an einer Krankheit leiden, die sich erfahrungsgemäss durch Kälte verschlechtert (z.B. höhergradige arterielle Durchblutungsstörungen). Dagegen wäre die «Schleimhautregie» auch für kranke Personen mit nur geringer Belastbarkeit geeignet; körperliche Aktivität allgemein kann individuell z.B. anhand des Trainingspulses dosiert und angepasst werden.

\section{Disclosure Statement}

There is no conflict of interest to declare.

\section{Literatur}

1 Zetkin M, Schaldach H (Hrsg): Stichwort «Abhärtung». Lexikon der Medizin, ed 16. München, Elsevier, 2006.

2 Brenke R, Bühring M: Abhärtung durch Hydrotherapie? Z Allg Med 1997;73:18-23.

3 Grober C, Riesberg A, Mansmann U, Knipschild P, Wahn U, Bühring M: The effect of hydrotherapy on the incidence of common cold episodes in children: a randomised clinical trial. Eur J Pediatr 2003;162:168-176.

4 Kanig F: Infektanfällige Kinder in der ambulanten physiotherapeutischen Betreuung (Erfahrungsbericht nach Elternbefragen). Z Phy siother 1988;40:229-235.

5 Kehnscherper M: Profilierung einer pädiatrischen Physiotherapie. Z Physiother 1990; 42:265-269.

6 Mikolásek A: Einige Beobachtungen über den Einfluss der Sauna auf den Gesundheitszustand bei Kindern. Sauna Arch 1969;7:1-8.

7 Ernst E, Wirz P, Pecho L: Wechselduschen und Sauna schützen vor Erkältung. Z Allg Med 1990;66:56-60.

8 Goedsche K, Förster M, Kroegel C, Uhlemann C: Serielle Kaltwasserreize (Kneipp'scher Ober guss) bei Patienten mit chronisch obstruktiver Bronchitis (COPD). Forsch Komplementär med 2007;14:158-166.

9 Menger W: Warum Abhärtung? Sozialpädiatrie 1980;2:243-249.

10 Menger W: Erfolge der Abhärtung bei Kin dern mit Asthma bronchiale. Pneumologie 1990;44:1183-1184.

11 Jacob EM, Volger E: Blutdrucksenkung durch Hydrotherapie: eine randomisierte, kontrollier te Studie bei leichter bis mittelschwerer Hypertonie. Phys Med Rehab Kuror 2009;19:162-168.

12 Brenke R: Winterschwimmen - eine Extremform des Abhärtungssports. Therapeutikon 1990;4:466-472.

13 Brenke R: Baden im eisigen Wasser. Über den gesundheitlichen Wert einer Extremform der Abhärtung. Saunabetrieb Bäderpraxis 1997; 8:20-26.

14 Conradi E, Brenke R, Philipp S: Häufigkeit res piratorischer Erkrankungen und sekretorisches Immunglobulin A im Speichel unter dem Einfluss regelmässigen Saunabadens von Kindern Z Phys Rehab Kur Med 1992;2:19-21.

15 Einenkel D: Verbesserung des Gesundheits zustandes von Kindergartenkindern im Kreis Annaberg durch den regelmässigen Besuch einer Betriebssauna. Z Ärztl Fortbild 1977;71:1069-1071.
16 Juhász J, Kunay M: Wirkung der finnischen Sauna bei Kindern mit rezidivierenden Erkrankungen der Atemwege und der Lungen. Sauna Arch 1969;7:8-17.

17 Eisermann P: Langzeitstudie zum regelmässigen Saunabaden einer Kindergruppe hinsichtlich thermischer Konditionierung. Berlin, Humboldt-Universität, Inauguraldissertation, 1985.

18 Philipp S, Brenke R, Conradi E, Krause H: Der Einfluss regelmässigen Saunabadens auf die lokale Abwehr des kindlichen Organismus gegenüber akuten respiratorischen Infektionen. Int Sauna Arch 1991;8:11-18.

19 Hartmann A: Die «asiatische» Grippe 1957 die Sauna als Prophylaktikum. Hippokrates 1958;29:153-154.

20 Fritzsche W: Sauna von A-Z, ed 4. Bielefeld, Sauna Matti, 1990.

21 Fritzsche W: Soziologische Untersuchungen zu Sauna-Verbreitung und Sauna-Gebrauch. Int Sauna Arch 1986;3:123-128.

22 Schaffranek L: Auswirkung der Sauna auf die Häufigkeit der Erkältungskrankheiten und die Arbeitsunfähigkeitsdauer. Sauna Arch 1968;6:23-25.

23 Cervello EM, Santos-Rosa FJ: Motivation in sport: an achievement goal perspective in young Spanish recreational athletes. Percept Mot Skills 2001;92:527-534.

24 Papaioannou A, Bebetsos E, Theodorakis Y, Christodoulidis T, Kouli O: Causal relationships of sport and exercise involvement with goal orientations, perceived competence and intrinsic motivation in physical education: a longitudinal study. J Sports Sci 2006;24:367382.

25 Schuh A: Möglichkeiten der modernen Klimatherapieformen. Phys Med Rehab Kur 1998;8:54-59.

26 Conradi E: Beitrag zum Anpassungsprozess des menschlichen Organismus an wiederholte thermische Belastungen. Berlin, HumboldtUniversität, Habilitationsschrift, 1980.

27 Hoffmann H: Die Beeinflussung der Hautdurchblutung, gemessen mittels der akralen Wiedererwärmungsreaktion durch eine Serie von Saunabädern unter Berücksichtigung des tageszeitlichen Verhaltens. Berlin, HumboldtUniversität, Inauguraldissertation, 1978.

28 Brenke R: Die Wirkung des Saunabades, Wärmephase und Abkühlung, auf die Durchblutung der Extremitäten. Int Sauna Arch 1990;7:161-164.
29 Brenke R, Siems W: Das Buch vom Winterschwimmen. Gesund und fit durch Abhärtung. Husum, Husum, 1996.

30 Lange Andersen K: Kälteanpassung bei Tieren und Menschen. Polarforschung 1960;30:11-18.

31 Brenke R, Brenke A, Conradi E: Verände rungen der Sublingualtemperatur während einer 5-wöchigen Saunaserie. Z Physiother 1979;31:389-390.

32 Brenke R: Der Wärmehaushalt des Men schen beim Saunabaden. Sauna Bäderpraxis 2007;18:19-23.

33 Heldmaier G: Vergleichende Tierphysiologie, ed 2. Vegetative Physiologie. Berlin, Springer 2004.

34 Brenke R: Die Sauna als Heilmittel. Naturheilkunde 2009;86:29-33.

35 Brenke R, Plew H, Warnke CK: Auswirkungen einer Serie von Kaltwasserbädern auf die ve getative Herznervensteuerung des Menschen. Z Physiother 1982;34:177-180.

36 Brenke R, Brenke A, Conradi E: Untersuchungen zur Frage des Langzeiteffektes unter ambulanter Bäderbehandlung. Z Physiother 1979;31:373-379.

37 Brenke R, Rathsack R, Conradi E, Krauss S: Die Beeinflussung des Tagesganges eines Neurotransmitters (Substanz P) - Grund für Schlafstörungen bei abendlicher Saunaanwendung? Int Sauna Arch 1986;3:75-76.

38 Brenke R, Conradi E: Erste Ergebnisse zur Wirkung intensiver Kälte (Eisbad) auf den Plasmaspiegel der Substanz P. Z Med Sport 1987;27:21-23.

39 Brenke R, Brenke A: Grundlagen für prophy laktische und therapeutische Wärme- und Kälteanwendungen bei Gesunden und Hautkran ken - dargestellt am Beispiel der progressiven Sklerodermie. Berlin, Humboldt-Universität, Habilitationsschrift, 1991.

40 Bieger WP, Penz M, Gruber R: Immunologie der Abhärtungsreaktion nach Hydrotherapie. Phys Rehab Kur Med 1998;8:37-45.

41 Kreutzfeld A, Albrecht B, Müller K: Einfluss des Wassertretens nach Kneipp auf die Immunregulation. Phys Med Rehab Kuror 2003;13:208-214.

42 Brenke R, Diezel W, Brenke A, Conradi E: Hy perthermie und Interferonsystem. Z Physiother 1983;35:193-194.

43 Brenke R: Neuere immunologische Befunde zur Erklärung der abhärtenden Wirkung der Sauna. Int Sauna Arch 1992;9:129-135.

44 Gastl G, Földinger A, Egg D, Herold M: Wirkung von Hyperthermie im Saunabad auf 
die natürliche Immunität. Int Sauna Arch 1985;2:5-7.

45 Brenke R, Müller GM, Materna A, Tanzmann $\mathrm{H}$ : Beeinflussung der Granulozytenfunktion durch Sauna - Hyperthermie. Z Klin Med 1987;42:2245-2248.

46 Materna A, Brenke R: Abhärtung durch Saunabaden. Therapeutikon 1991;5:433-437.

47 Rysánková J: Mucociliar transport of nasal mucosa during sauna bathing. Int Sauna Arch 1988;5:137-139.

48 Brenke R, Conradi E, Krause H, Porstmann B: Lokale Immunabwehr und Sauna, dargestellt an der Immunglobulin-A-Konzentration im Speichel. Int Sauna Arch 1985;2:1-4.

49 Philipp S, Brenke R, Conradi E, Krause H: Der Einfluss regelmässigen Saunabadens auf die lokale Abwehr des kindlichen Organismus gegenüber akuten respiratorischen Infektionen. Int Sauna Arch 1991;8:11-18.

50 Brenke R, Siems W: Klinisch-chemische Untersuchungen beim Eisbaden - Hinweise für einen oxidativen Stress. Z Physiother 1991;43:93-102.
1 Brenke R, Siems W, Maass R: Abhärtung durch Kaltreize unterschiedlicher Intensität: Wirkungen auf den Purin- und Radikalmetabolismus. Wien Med Wochenschr 1994;144:66-68.

52 Grünberger P, Siems WG, Brenke R, Jakstadt M, Grune T, Conradi E: Vitamin C and uric acid decreases during short-term whole body cold exposure for disease prevention (abstract); in Krinsky NI, Sies H (eds): Antioxydant Vitamins and Beta-Carotene in Disease Prevention. Berlin, Society for Free Radical Research, 1994, p 89.

53 Siems W, Brenke R: Changes in the glutathione system of erythrocytes due to enhanced formation of oxygen free radicals during short-term whole body cold stimulus. Arctic Med Res 1992;51:3-9.

54 Siems WG, van Kuijk FJGM, Maass R, Brenke R: Uric acid and glutathione levels during short-term whole body cold exposure. Free Radic Biol Med 1994;16:299-305.

55 Siems WG, van Kuijk FJGM, Maass R, Grune T, Brenke R: Uric acid, glutathione and vitamin
C levels during short-term whole body cold exposure (abstract); in Xin W, Packer L, Gu F (eds): Natural Antioxidants - Molecular Mechanisms and Health Effects. Beijing, Academia Sinica, 1995, p 320.

56 Siems WG, Brenke R, Sommerburg O, Grune $\mathrm{T}$ : Improved antioxidative protection in winter swimmers. QJM 1999;92:193-198.

57 Conradi E, Brenke R, Grune T, Grünberger P, Kästner P, Kästner A: Beeinflussung des Radikal-Stoffwechsels durch Saunawärme und kurzzeitige Abkühlung. Int Sauna Arch 1994;11:55-59.

58 Eisermann P: Effekte der Kurorttherapie auf die periphere Mikrozirkulation anhand der Messung der akralen Wiedererwärmungszeit. Vortrag. XIII. Kongress der Gesellschaft für Physiotherapie, Bad Elster, 4.-8. Dezember 1988.

59 Brenke R, Bühring M: Abhärtung durch Hydrotherapie? Z Allg Med 1997;73:18-23. 